\title{
Improving Outcomes After Distal Pancreatectomy with Celiac Axis Resection (DP-CAR): As Always, it is All About Patient Selection
}

\author{
Naruhiko Ikoma, MD, MS and Matthew H. G. Katz, MD \\ Department of Surgical Oncology, MD Anderson Cancer Center, University of Texas, Houston, TX
}

Resection of the celiac axis and pancreatic tail in an attempt to improve the survival of patients with gastric cancer by increasing the radicality of gastrectomy to include adjacent blood vessels and lymph nodes was originally described in $1953 .{ }^{1}$ A "modified Appleby procedure" (distal pancreatectomy with celiac axis resection [DP-CAR]) was subsequently developed to resect pancreatic tumors inseparable from the celiac trunk. ${ }^{2}$ Although the eagerness of surgeons to perform this operation for advanced pancreatic cancer has only grown since then, data accumulated primarily from small, single-institution reports have generally illustrated an operation associated with a high rate of morbidity and a low rate of cure. In this context, Dr. Klompmaker and colleagues have analyzed the combined experience of surgeons on three continents who performed DP-CAR during the past two decades in order to identify clinical features shared by patients most likely to benefit from it. ${ }^{3}$ Do data from this vast experience justify the enthusiasm with which DP-CAR is so often performed?

This study showed what appears, at least on the face of it, to be a critical relationship between DP-CAR volume and perioperative mortality. In this multi-institutional experience, $18 \%$ of the patients who underwent DP-CAR at a low-volume center died within 90 days after surgery, compared with only $5.5 \%$ of the patients at a high-volume center. However, a high-volume center was defined as one at which only one DP-CAR was performed annually within a recent 3-year period. Fundamentally, this low limit for

(C) Society of Surgical Oncology 2018

First Received: 5 November 2018; Published Online: 11 December 2018

M. H. G. Katz, MD

e-mail:mhgkatz@mdanderson.org high DP-CAR volume highlights the infrequency with which the celiac trunk is encased by cancers that are otherwise resectable, and for which surgery is even remotely appropriate oncologically.

That a significant difference in perioperative mortality was associated with a surgical volume as low as a single operation per year also suggests that the favorable morbidity rate shared by institutions with more frequent performance of DP-CAR is largely due to something other than wisdom or technical skill that can be accumulated simply by performing more DP-CAR operations. Furthermore, although the wisdom and technical skill presumably necessary to conduct DP-CAR safely might be assumed to come with accumulated experience performing pancreatic surgery more generally, the overall 90 -day mortality rate after DP-CAR performed at institutions included in the design cohort here, all considered to be high-volume centers for pancreas surgery (median, 70 pancreatoduodenectomies annually), was $16 \%{ }^{4}$ This is all to say that while we certainly agree with Klompmaker and colleagues that DP-CAR can be performed safely, the operation carries a real risk for perioperative morbidity and mortality, irrespective of where it is performed or how experienced the surgical team performing it may be.

In this regard, Klompmaker and colleagues should be applauded for their attempt to identify high-risk patients unlikely to benefit from DP-CAR. However, the short-term metric of perioperative morbidity they primarily analyzed represents a grossly incomplete basis for decision-making for patients with a disease in which occult systemic disease leads to incurable cancer recurrence and death after $80 \%$ of pancreatectomies. With respect to the role for this potentially morbid operation in the treatment of pancreatic 
cancer, could Dr. Blake Cady's ${ }^{5}$ admonition that "biology is king, selection of cases is queen" be any more important?

When DP-CAR (or really, any operation) is considered for pancreatic cancer, the known risks of the operation must be aligned with a realistic assessment of the potential for long-term survival. Clinical data such as symptoms, performance status, radiographic stage, and serum CA 19-9 level $^{6}$ can and should be used to develop a composite impression of the burden of systemic disease that may left behind, and ultimately may lead to death, after even the most technically flawless of operations. In the near future, assays of systemic disease that quantify circulating tumor cells, nucleic acids or exosomes, ${ }^{7}$ or imaging biomarkers ${ }^{8}$ may provide a more objective estimate of the potential for long-term benefit after aggressive local therapies such as DP-CAR.

At the University of Texas MD Anderson Cancer Center, at two of the three very-high-volume institutions in the validation cohort of this study, and increasingly at pancreatic treatment centers nationwide, preoperative therapy has been recognized as an effective means for selecting patients to undergo pancreatectomy. ${ }^{9}$ We typically treat any patient anticipated to require venous and/or arterial resection with a prolonged course of systemic chemotherapy and consider subsequent radiation in selected cases. ${ }^{10}$ We stage each with cross-sectional imaging, a physical examination, and an assessment of CA 19-9 every 2 months during preoperative therapy and encourage exercise to improve physiologic status. ${ }^{11} \mathrm{We}$ consider robust patients who exhibit no evidence of metastatic progression (those selected to have a "locally dominant phenotype") as ideal candidates for pancreatectomy. ${ }^{12}$

Indeed, although the enthusiasm of surgeons to perform DP-CAR seems unlimited, we strongly believe that the key to improving its outcomes for patients with cancer is to limit its use, not simply to those for whom the likelihood of perioperative morbidity may be low, but to the select few for whom long-term survival also can be anticipated.

\section{REFERENCES}

1. Appleby LH. The coeliac axis in the expansion of the operation for gastric carcinoma. Cancer. 1953;6:704-7.

2. Klompmaker S, de Rooij T, Korteweg JJ, et al. Systematic review of outcomes after distal pancreatectomy with coeliac axis resection for locally advanced pancreatic cancer. $\mathrm{Br} J$ Surg. 2016;103:941-9.

3. Klompmaker S, Peters N, van Hilst J, et al. Outcomes and risk score for distal pancreatectomy with celiac axis resection (DPCAR): an international multicenter analysis. Ann Surg Oncol. 2018. https://doi.org/10.1245/s10434-018-07101-0.

4. Klompmaker S, van Hilst J, Gerritsen SL, et al. Outcomes after distal pancreatectomy with celiac axis resection for pancreatic cancer: a pan-European retrospective cohort study. Ann Surg Oncol. 2018;25:1440-7.

5. Cady B. Basic principles in surgical oncology. Arch Surg. 1997;132:338-46.

6. Bergquist JR, Puig CA, Shubert CR, et al. Carbohydrate antigen 19-9 elevation in anatomically resectable, early-stage pancreatic cancer is independently associated with decreased overall survival and an indication for neoadjuvant therapy: a national cancer database study. J Am Coll Surg. 2016;223:52-65.

7. Bernard V, Kim DU, San Lucas FA, et al. Circulating nucleic acids are associated with outcomes of patients with pancreatic cancer. Gastroenterology. 2018. https://doi.org/10.1053/j.gastro. 2018.09.022.

8. Koay EJ, Lee Y, Cristini V, et al. A visually apparent and quantifiable CT imaging feature identifies biophysical subtypes of pancreatic ductal adenocarcinoma. Clin Cancer Res. 2018;24:5883-94.

9. Katz MH, Shi Q, Ahmad SA, et al. Preoperative modified FOLFIRINOX treatment followed by capecitabine-based chemoradiation for borderline resectable pancreatic cancer: Alliance for Clinical Trials in Oncology trial A021101. JAMA Surg. 2016;151:e161137.

10. Katz MHG, Ou FS, Herman JM, et al. Alliance for clinical trials in oncology (ALLIANCE) trial A021501: preoperative extended chemotherapy vs chemotherapy plus hypofractionated radiation therapy for borderline resectable adenocarcinoma of the head of the pancreas. BMC Cancer. 2017;17:505.

11. Parker NH, Ngo-Huang A, Lee RE, et al. Physical activity and exercise during preoperative pancreatic cancer treatment. Support Care Cancer. 2018. https://doi.org/10.1007/s00520-018-4493-6.

12. Cloyd JM, Katz MH, Prakash L, et al. Preoperative therapy and pancreatoduodenectomy for pancreatic ductal adenocarcinoma: a 25-year single-institution experience. $J$ Gastrointest Surg. 2017;21:164-74. 